\title{
Why not to publish Case Reports?
}

\author{
The Editorial Board
}

$\mathrm{A}$ Case Report is defined as a description of the clinical signs, symptoms, diagnosis, treatment, and follow-up of an individual patient with some unusual features that makes it interesting for the target readership of a scientific journal, followed by a brief discussion. Clinical and Translational Oncology has published Case Reports since its launch in 2005 as a service to the oncology community. Indeed, Case Reports had their moment and opportunity and covered an important need for oncology clinicians for all these years.

Recently, The Editorial Board of Clinical and Translational Oncology decided to discontinue its policy of publishing Case Reports. Several major reasons are the basis for this change. First, our journal is progressively becoming more specialized in the publication of updated reviews on all aspects of translational oncology, as well as consensus and guidelines useful to support the day-to-day needs of clinicians practicing state-of-the-art clinical oncology. Five different, complementary series now comprise our goal, with the aim of covering a large spectrum of themes that will satisfy the needs of our wide readership, which consists of both basic researchers and clinical oncologists within four of its major specialties: medical oncology, radiation oncology, surgical oncology, and pediatric oncology. Our major commitment has been, and will continue to be, to foster communication among experimental and clinical oncologists by providing the arena in which to meet and discuss novel and promising translational approaches. This is a very ambitious commitment that requires great effort and dedication, along with appropriate formats for their presentation.

Clinical and Translational Oncology also intends to continue reporting original scientific studies at the basic,

translational, and clinical oncology levels. This is always a great complement to the updated information that oncologists need and a very attractive spotlight for all readers. Furthermore, original research is the very heart of the attitude toward this devastating disease. Still unexplored avenues must be taken in the attempt to defeat cancer, and original and novel research is the best approach. Our journal is devoted to serving as the vehicle by which to convey the need for translational oncology - the best way to close the gap between progress being made in understanding the molecular mechanisms behind the origin of cancer and the necessity for improved clinical management of cancer patients.

Finally, but still important, space in specialized oncology journals is becoming a limited commodity. Both experimental and clinical oncologists have limited time to dedicate to being updated in their respective areas of expertise. Vigorous competition among journals to be essential sources of the most reliable and useful information is becoming extremely important. This competition necessitates all scientific journals to be more selective with what they offer their readership. Space limitation is therefore an unavoidable imposition, and our journal cannot ignore this obligation to our readers.

Within this context, Case Reports provide limited contributions to the goals of our journal. In the following months, only Case Reports already accepted will be published, and further Case Reports will no longer be accepted.

We firmly believe this decision will improve our attempts to focus on the needs of our readers and help further advance our intention that Clinical and Translational Oncology continues to be a high-quality working instrument for oncologists. 